Como citar este artigo

Couto SMF, Brandi BA, Souza AA, Silva VC, Conde C, Machado DI, Vattimo MFF. [Avaliação

de um programa de exercício físico moderado no tratamento de Doença

Renal Crônica: estudo

pré-clínico]. Rev Paul Enferm [Internet]. 2019;30 doi:10.33159/25959484 repen.2019v30a7

\title{
Avaliação de um programa de exercício físico moderado no tratamento de Doença Renal Crônica: estudo pré-clínico
}

\author{
Evaluation of a Moderate Exercise Program in the Treatment of Chronic \\ Kidney Disease: a Preclinical Study \\ Evaluación De Un Programa De Ejercicio Físico Moderado En El Tratamiento \\ De La Enfermedad Renal Crónica: Estudio Pré-Clínico
}

Sheila Marques Fernandes CoutoI, Beatriz Almeida BrandiI, Adriana Almeida de Souza"II, Vinicius Cardoso da Silva ${ }^{\mathrm{III}}$, Carolina Conde ${ }^{\mathrm{III}}$, Douglas Ikedo Machado'II, Maria de Fatima Fernandes Vattimo ${ }^{\text {IV }}$

I Mestre em ciências. Universidade de São Paulo, Escola de Enfermagem, Laboratório Experimental de Modelos Animais, São Paulo, SP, Brasil.

II Mestranda em ciências. Universidade de São Paulo, Escola de Enfermagem, Laboratório Experimental de Modelos Animais, São Paulo, SP, Brasil.

III Iniciação científica. Universidade de São Paulo, Escola de Enfermagem, Laboratório Experimental de Modelos Animais, São Paulo, SP, Brasil.

IV Livre-Docente. Universidade de São Paulo, Escola de Enfermagem, Laboratório Experimental de Modelos Animais, São Paulo, SP, Brasil.

\section{RESUMO}

INTRODUÇÃO: A Doença Renal Crônica é um problema de saúde pública. O exercício físico aeróbio em intensidade moderada pode prevenir ou retardar a progressão da Doença Renal Crônica. OBJETIVO: Avaliar a função renal e a hemodinâmica renal de ratos com doença renal crônica submetidos ao exercício físico aeróbio de intensidade moderada. MÉTODOS: Ratos, adultos, distribuídos nos grupos: Sham (controle cirúrgico); Sham + Exercício físico; Doença renal crônica; Doença renal crônica + Exercício físico. O Exercício físico consistiu em natação diária, 5 dias/semana, 60 minutos/dia, com carga adicional colocada na calda correspondente a $5 \%$ do peso corporal. Foram avaliadas a função renal (clearance de inulina, creatinina sérica) e a hemodinâmica renal (fluxo sanguíneo renal e resistência vascular renal). RESULTADOS: No grupo Doença renal crônica houve diminuição do clearance de inulina e do fluxo sanguíneo renal com elevação na creatinina sérica e da resistência vascular renal em relação aos grupos Sham e Sham + Exercício físico. O grupo Doença renal crônica + Exercício físico demonstrou melhora desses parâmetros quando comparados ao grupo Doença renal crônico não exercitado. CONCLUSÃO: O Exercício físico em intensidade moderada promoveu melhora da função renal com recuperação da hemodinâmica renal na doença renal crônica.

Descritores: Doença renal crônica; Exercício físico aeróbico; Exercício físico de intensidade moderada; Terapias Complementares; Experimentação Animal. 


\section{ABSTRACT}

Background: Chronic kidney disease is a public health problem. Moderate-intensity aerobic exercise can prevent or slow the progression of chronic kidney disease. Aims: To evaluate the renal function and the renal hemodynamics in rats with chronic kidney disease undergoing moderate-intensity aerobic exercise. Methods: Adult rats were distributed in the following groups: sham (surgical control); sham + physical exercise; chronic kidney disease; chronic kidney disease + physical exercise. Physical exercise consisted of daily swimming, five days/week, 60 minutes/day, with an additional load placed in the tail corresponding to $5 \%$ of the animal's body weight. Renal function (inulin clearance, serum creatinine), and renal hemodynamics (renal blood flow and renal vascular resistance) were evaluated. Results: There was a decrease in inulin clearance and renal blood flow, accompanied by an increase in serum creatinine and renal vascular resistance in the chronic kidney disease group compared to the sham and sham + exercise groups. The chronic kidney disease + exercise group showed improvement in these parameters compared to the chronic kidney disease group with no exercise. Conclusion: Moderate-intensity exercise resulted in the improvement of renal function and renal hemodynamic recovery in rats with chronic kidney disease. Keywords: Chronic kidney disease; Aerobic exercise; Moderate-intensity physical exercise; Complementary Therapies; Animal Experimentation.

\section{RESUMEN}

INTRODUCCIÓN: La Enfermedad Renal Crónica es un problema de salud pública. El ejercicio físico aeróbico en intensidad moderada puede prevenir o retrasar la progresión de la enfermedad renal crónica. OBJETIVO: Evaluar la función renal y la hemodinámica renal en ratones con enfermedad renal crónica sometidos a ejercicio aeróbico de intensidad moderada. MÉTODOS: Ratones, adultos, distribuidos en grupos: Sham (control quirúrgico); Sham + ejercicio físico; Enfermedad renal crónica; Enfermedad renal crónica + ejercicio físico. El ejercicio físico consistió en natación diaria, 5 días / semana, 60 minutos / día, con carga adicional colocada en el jarabe correspondiente al $5 \%$ del peso corporal. Se evaluaron la función renal (clearance de inulina, creatinina sérica) y la hemodinámica renal (flujo sanguíneo renal y resistencia vascular renal). Resultados: En el grupo de Enfermedad renal crónica hubo una disminución en el clearance de inulina y del flujo sanguíneo renal con un aumento en la creatinina sérica y la resistencia vascular renal en comparación con los grupos de ejercicio Sham y Sham +. El grupo de enfermedad renal crónica + ejercicio mostró una mejora en estos parámetros en comparación con el grupo de enfermedad renal crónica no ejercitado. CONCLUSIÓN: El ejercicio en intensidad moderada promovió una mejora de la función renal con recuperación de la hemodinámica renal en la enfermedad renal crónica.

Descriptores: enfermedad renal crónica; Ejercicio aeróbico; Ejercicio físico de intensidad moderada; Terapias complementarias; Experimentación Animal.

\section{INTRODUÇÃO}

Decorrente de nefropatias ou complicações do Diabetes Mellitus e hipertensão predominantemente, a Doença renal crônica (DRC) é considerada um problema de saúde pública por sua elevada taxa de morbi-mortalidade e redução da qualidade de vida ${ }^{(1)}$. A DRC consiste em síndrome que afeta os rins de maneira progressiva e irreversível causando redução da filtração glomerular com comprometimento da manutenção da hemóstase do organismo(2). Tratamentos paliativos de terapia de substituição renal, como a diálise ou transplante renal, possibilitam o prolongamento da sobrevida, mas a epidemiologia dessa síndrome sinaliza para a necessidade de adoção estratégias preventivas para controle da progressão da $\mathrm{DRC}(3)$.

Dados do Censo da Sociedade Brasileira de Nefrologia publicados no mais recente Inquérito Brasileiro de Diálise Crônica demonstram que 12.583 pacientes realizaram tratamento dialítico no ano de 2017 e destacam a alta prevalência da DRC-(4). As estatísticas apresentadas 
salientam ainda a elevada mortalidade de 25.187 óbitos de pacientes em diálise em 2017, contra 31.226 pacientes na lista de transplantes renais, tratamento considerado como melhor relação custo-benefício para a $\mathrm{DRC}\left({ }^{(4)}\right.$.

A progressão da DRC para desfechos indesejados pode ser prevenida ou retardada por meio de medidas que favoreçam o diagnóstico precoce e implementação de medidas nefroprotetoras ${ }^{(5)}$. A falta de conhecimento da definição e estadiamento da doença juntamente com o uso de testes tardios para avaliação da doença acarretam no subdiagnóstico e tratamentos inadequados que resultam na perda de oportunidade para a implementação de estratégias de prevenção(6).

A prática de exercício físico (EF) regular, uma estratégia de prevenção primária, é referida como uma intervenção não farmacológica no tratamento de diversas doenças com promoção de benefícios fisiológicos, cognitivos, psicoemocionais e sociais, influenciando diretamente a melhora da qualidade de vida ${ }^{(7)}$.

Indivíduos com DRC apresentam uma redução da capacidade física e funcional que favorece o sedentarismo, atrofia muscular, anemia, entre outras comorbidades associadas ${ }^{(8)}$. Neste contexto, um programa de EF regulares pode promover adaptações envolvidas na vasoconstrição renal por envolvimento do estimulo do sistema nervoso simpático e controle hormonal que permite uma resposta hemodinâmica que melhora a homeostase (9).

A proposição de programas de EF que visem o tratamento dos sinais clínicos da DRC e sua repercussão na melhora na qualidade de vida devem ser investigados e encorajados pela comunidade científica e profissionais de saúde. Estudos que permitam o isolamento de variáveis possibilitam a avaliação e quantificação de resposta fisiológicas ao exercício físico aeróbico moderado fornecendo embasamento científico para prescrição e orientação segura dessa prática por profissionais de saúde, como enfermeiros que acompanham pacientes com DRC, configurando-se como uma estratégia renoprotetora na progressão para doença renal crônica terminal.

O presente estudo se propõe elucidar as alterações funcionais e hemodinâmicas envolvidas na fisiopatologia do DRC e na prática de EF utilizando um modelo experimental pré-clínico sob hipótese de que o programa de EF em intensidade moderada possa ter efeito nefroprotetor na DRC. Portanto, o objetivo deste estudo foi avaliar a função e hemodinâmica renal de ratos com DRC submetidos a treinamento físico em intensidade moderada.

\section{MÉTODO}

\section{Tipo de estudo}

Estudo pré-clínico quantitativo experimental com modelo animal, utilizando ratos da raça Wistar, machos, adultos, pesando entre 250-350 gramas, fornecidos pelo Instituto de Ciências Biomédicas (ICB-USP).

\section{Cenário}

O estudo foi desenvolvido no Laboratório experimental de Modelos Animais (LEMA) da Escola de Enfermagem da Universidade de São Paulo (EEUSP).

\section{Coleta de dados}

A coleta e análise dos dados para este estudo foram realizadas no período de fevereiro de 2018 a fevereiro de 2019. 
Animais: Foram utilizados 20 ratos da raça Wistar, machos, adultos, pesando entre 250 e 350 gramas. Os animais foram distribuídos nos seguintes grupos: Sham (controle cirúrgico, $\mathrm{n}=5$ ): ratos submetidos a simulação do ato cirúrgico do modelo de lesão renal crônica sem alteração das estruturas renais; Sham+Exercício físico (Sham+EF, $n=5$ ): ratos submetidos a simulação do ato cirúrgico do modelo de lesão renal crônica sem alteração das estruturas renais e após 3 dias de recuperação cirúrgica, foram iniciados os treinamentos físicos 60 minutos diários, 5 dias consecutivos, por 25 dias; $\mathrm{DRC}(\mathrm{n}=5)$ : ratos submetidos à técnica $5 / 6$; $\mathrm{DRC}+$ Exercício físico $(\mathrm{DRC}+\mathrm{EF}, \mathrm{n}=5)$ : ratos submetidos à técnica $5 / 6$ de ablação de massa renal e, após 3 dias de recuperação cirúrgica, foram iniciados os treinamentos físicos, 60 minutos diários, 5 dias consecutivos, por 25 dias. A intensidade moderada do EF foi estabelecida pela colocação de carga equivalente a $4 \%$ do peso corporal do animal junto a calda do mesmo durante as sessões de natação(10).

Obtenção do material biológico: Ao final dos protocolos experimentais de cada grupo, os animais foram colocados em gaiolas metabólicas para coleta de urina de 24 horas, para estudos da função renal. Posteriormente, os animais foram anestesiados com Thiopentax ${ }^{\circledR}$ (tiopental sódico $40-50 \mathrm{mg} / \mathrm{kg}$, via intraperitoneal- i.p.) e submetidos à laparotomia para a mensuração do fluxo sanguíneo renal (FSR) na artéria renal esquerda, que foi isolada e envolvida por sonda ultrassônica (T402; Transonic Systems, Bethesda, Maryland, EUA), avaliação da pressão arterial média (PAM) invasiva após dissecção e cateterização da artéria carótida para inserção de cateter (tubo de polietileno - PE 60), coleta de sangue terminal por punção da aorta abdominal(11).

Função renal: A função renal foi avaliada pelo clearance de inulina e creatinina sérica. A inulina sérica e urinária foi quantificada pelo método de Antrona ${ }^{(12)}$. A creatinina sérica foi mensurada pelo método de Jaffé ${ }^{(13)}$.

Hemodinâmica renal: A hemodinâmica renal foi mensurada pelo FSR e resistência vascular renal (RVR), considerando a PAM. Para cálculo da RVR, utilizou-se da seguinte fórmula: $\mathrm{RVR}=\mathrm{PAM} / \mathrm{FSR}^{(11,12)}$.

\section{Análise e tratamento dos dados}

Os resultados estão apresentados como média \pm desvio-padrão. $O$ tratamento dos dados foi feito por meio da análise de variância ANOVA, seguida do pós-teste de Newman-Keuls Multiple para comparações entre os grupos, usando GraphPad Prism 6.01® software. O $p<0,05$ foi considerado como significante.

\section{Aspectos éticos}

O estudo foi aprovado pela Comissão de Ética em Experimentação Animal da Faculdade de Medicina da Universidade de São Paulo (CEUAS- FMUSP) sob o protocolo n. ${ }^{\circ}$ 1073/2018. Os procedimentos realizados no presente estudo estão de acordo com os princípios éticos de experimentação animais adotados pelo Colégio Brasileiro de Experimentação Animal (COBEA).

\section{RESULTADOS}

\section{Função Renal}

Como demonstrado na Tabela 1, os animais DRC apresentaram discreta elevação dos parâmetros fluxo urinário e creatinina sérica em relação aos animais dos grupos Sham e Sham+E. Os animais do grupo DRC+E apresentaram manutenção no fluxo urinário e redução da creatinina sérica comparados com animais do grupo DRC. 
Na análise do clearance de inulina, observou-se uma redução nos grupos DRC e DRC+E comparado aos animais Sham e Sham+E. O grupo DRC+E apresentou elevação do clearance de inulina comparado ao DRC.

Tabela 1. Função renal dos grupos Sham, Sham+E, DRC e DRC+E. São Paulo 2019.

\begin{tabular}{|l|c|c|c|c|}
\hline Grupos & $\mathbf{n}$ & $\begin{array}{c}\text { Fluxo urinário } \\
(\mathbf{m l} / \mathbf{m i n})\end{array}$ & $\begin{array}{c}\text { Creatinina Sérica } \\
\mathbf{( m g / d l )}\end{array}$ & $\begin{array}{c}\text { Clearance de inulina } \\
\mathbf{( m l / m i n})\end{array}$ \\
\hline Sham & 5 & $0,014 \pm 0,002$ & $0,20 \pm 0,01$ & $0,70 \pm 0,03$ \\
\hline Sham+EF & 5 & $0,013 \pm 0,002$ & $0,21 \pm 0,06$ & $0,72 \pm 0,06$ \\
\hline DRC & 5 & $0,022 \pm 0,004^{\mathrm{ab}}$ & $0,91 \pm 0,09^{\mathrm{ab}}$ & $0,26 \pm 0,03^{\mathrm{ab}}$ \\
\hline DRC+EF & 5 & $0,021 \pm 0,002^{\mathrm{ab}}$ & $0,43 \pm 0,06^{\mathrm{abc}}$ & $0,41 \pm 0,03^{\mathrm{abc}}$ \\
\hline
\end{tabular}

DRC: Doença renal crônica. EF: Exercício físico. ${ }^{a} p<0,001$ vs Sham; ${ }^{b} p<0,001$ vs Sham+EF; ${ }^{c} p<0,001$ vs DRC

\section{Hemodinâmica Renal}

A PAM mostrou elevação significativa no grupo DRC em relação aos grupos controles saudáveis Sham e Sham+E. A prática de EF no DRC+EF demonstrou reduzir a PAM dos animais com DRC. O FSR foi reduzido nos animais DRC comparado aos grupos Sham e Sham+E, enquanto o grupo DRC+E demonstrou uma elevação do FSR comparado aos animais DRC que não foram submetidos ao treinamento físico. A RVR dos animais DRC e DRC+E aumentou em relação ao grupo Sham e Sham $+E$, entretanto, o grupo $D R C+E$ apresentou uma redução da RVR quando comparado ao grupo DRC.

Tabela 2. Hemodinâmica renal dos grupos Sham, Sham+E, DRC e DRC+E. São Paulo 2019.

\begin{tabular}{|l|c|c|c|c|}
\hline Grupos & $\mathbf{N}$ & $\begin{array}{c}\text { PAM } \\
(\mathbf{m m H g})\end{array}$ & $\begin{array}{c}\text { FSR } \\
(\mathbf{m l} / \mathbf{m i n})\end{array}$ & $\begin{array}{c}\mathbf{R V R} \\
(\mathbf{m m H g} / \mathbf{m l} / \mathbf{m i n})\end{array}$ \\
\hline Sham & 5 & $99,00 \pm 11,33$ & $9,28 \pm 1,73$ & $11,15 \pm 1,62$ \\
\hline Sham $+\mathrm{F}$ & 5 & $96,25 \pm 6,55$ & $8,10 \pm 0,14$ & $11,44 \pm 0,53$ \\
\hline DRC & 5 & $144,40 \pm 7,89^{\mathrm{ab}}$ & $5,76 \pm 0,35^{\mathrm{ab}}$ & $22,57 \pm 2,30^{\mathrm{ab}}$ \\
\hline DRC+EF & 5 & $99,67 \pm 11,66^{\mathrm{c}}$ & $7,98 \pm 1,41^{\mathrm{c}}$ & $17,01 \pm 0,48^{\mathrm{abc}}$ \\
\hline
\end{tabular}

DRC: Doença renal crônica. EF: Exercício físico. ${ }^{a} p<0,001$ vs Sham; ${ }^{b} p<0,001$ vs Sham $+E F ;{ }^{c} p<0,001$ vs DRC.

\section{DISCUSSÃO}

Definida clinicamente como taxa de filtração glomerular (TFG) inferior $60 \mathrm{~mL} / \mathrm{min} / 1,73 \mathrm{~m} 2$, resultante de uma lesão renal, por pelo menos três meses, a DRC é classificada em cinco estágios evolutivos de acordo com as alterações na TFG, sendo o quinto estágio caracterizado pelo comprometimento severo da função renal(14).

A fisiopatologia da DRC é complexa e está relacionada a existência de condição inflamatória que favorece a progressão da lesão renal. Essa condição se desenvolve a partir da lesão glomerular persistente que induz instalação de hipertensão glomerular e aumento da filtração glomerular resultando em proteinúria. A proteinúria está relacionada à hipertensão glomerular e ao aumento da liberação de mediadores inflamatórios que favorecem ao acúmulo de células no interstício renal. Em resposta a este processo inflamatório, ocorrem alterações na membrana basal glomerular e fibrose túbulo intersticial que resultam em perda progressiva da filtração 
glomerular ${ }^{(15,16)}$. Glomerulonefrites, hipertensão arterial, Diabetes Mellitus, nefropatias hereditárias, doenças sistêmicas e nefrites intersticiais crônicas são apontadas como as principais causas de DRC, sendo que no Brasil prevalecem a hipertensão arterial e o diabetes mellitus(17).

Os principais tratamentos para a doença renal crônica são o tratamento conservador, o qual inclui o controle adequado da pressão arterial; controle adequado da glicemia; interrupção do tabagismo; tratamento da dislipidemia; uso de medicamentos para diminuir a perda de proteínas pelos rins; tratamento da anemia, dos distúrbios ósseos e minerais, da acidose, da hipercalemia e dieta adequada. Na indicação de diálise, casos de pacientes com função renal residual abaixo de $20 \%$, são indicados o preparo do paciente para a terapia de substituição da função renal por diálise ou transplante ${ }^{(18)}$.

O estímulo ao exercício físico consiste em uma das intervenções preconizadas pela Organização Mundial de Saúde (OMS) para a redução da mortalidade prematura relacionada às doenças crônicas não transmissíveis ${ }^{(19)}$. Já foi demonstrado que o exercício físico moderado com treinamento aeróbio pode levar a adaptações renais de repouso, resultando em meIhora da filtração glomerular e melhora da reabsorção renal de sódio e potássio, no entanto, poucos estudos reproduziram, até momento, o modelo experimental de DRC com animais e o impacto de um programa de EF de intensidade moderada(20).

Os resultados do presente estudo confirmam a reprodução do modelo experimental de DRC com comprometimento da função e hemodinâmica renal sugerindo o potencial benefício da prática de um treinamento físico de intensidade moderada para a manutenção da funcionalidade e condições hemodinâmicas renais.

A redução da quantidade de néfrons obtida nefrectomia $5 / 6$ no modelo experimental de DRC inicialmente resulta em um quadro de lesão renal aguda com hiperfiltração como um mecanismo adaptativo. A evolução da DRC resulta no declínio progressivo da função renal conforme demonstrado no presente estudo, com aumento na creatinina plasmática e diminuição do clearance de inulina.

A DRC provoca alterações na hemodinâmica renal com aumento da resistência vascular renal e consequente diminuição do fluxo sanguíneo renal. Essas alterações podem estar relacionadas ao mecanismo adaptativo de hiperfiltração que resulta em alterações hemodinâmicas por ativar a cascata da renina que, por sua vez, induz à hipertensão glomerular, glomerulosclerose e deterioração da função renal(21).

A melhora hemodinâmica na DRC precede a melhora da função neste estudo e destaca a eficácia do programa de EF de intensidade moderada na prevenção da progressão da DRC. O EF confirma-se como intervenção não farmacológica na prevenção da progressão da DRC.

\section{CONCLUSÃO}

A indução de DRC reduziu a função renal dos ratos com envolvimento de alterações hemodinâmicas. O EF em animais DRC promoveu ação benéfica à função com melhora da hemodinâmica renal.

\section{Generalizações e limitações do estudo}

Esse estudo em modelo animal permitiu isolar variáveis não abordáveis em humanos para identificar respostas fisiológicas da prática de exercício físico aeróbico de intensidade moderada no DRC e fornecer embasamento científico para prescrição de um programa de treinamento físico. As limitações desse estudo consistem na ausência de estadiamento do modelo de DRC em animais, protocolos curtos de acompanhamento dos animais e necessidade de adequação do treinamento físico para transposição para prática clínica. 


\section{REFERÊNCIAS}

1. Levey AS, Astor BC, Stevens LA, Coresh J. Chronic kidney disease, diabetes, and hypertension: what's in a name? Kidney Int. $2010 ; 78(1): 19-22$.

2. Romão Junior JE. Doença Renal Crônica: Definição, Epidemiologia e Classificação. Braz. J. Nephrol. 2004;26(3 Suppl 1):1-3

3. Pecoits-Filho R, Rosa-Diez G, Gonzalez-Bedat M, Marinovich S, Fernandez S, Lugon J et al. Tratamento substitutivo da função renal na doença renal crônica: uma atualização do Registro Latino-Americano de Diálise e Transplante. J Bras Nefrol. 2015;37(1):9-13.

4. Thomé FS, Sesso RC, Lopes AA, Lugon JR, Martins CT. Inquérito brasileiro de diálise crônica 2017. J Bras Nefrol. 2019;41(2):208-14.

5. Bastos MG, Kirsztajn GM. Doença renal crônica: importância do diagnóstico precoce, encaminhamento imediato e abordagem interdisciplinar estruturada para melhora do desfecho em pacientes ainda não submetidos à diálise. J Bras Nefrol. 2011;33(1):93-108.

6. Sarmento LR, Fernandes PFCBC, Pontes MX, Correia DBS, Chaves VCB, Carvalho CFA et al. Prevalência das causas primárias de doença renal crônica terminal (DRCT) validadas clinicamente em uma capital do Nordeste brasileiro. J. Bras. Nefrol. 2018;40(2):130-5.

7. Coelho CFaria, Burini RC. Atividade física para prevenção e tratamento das doenças crônicas não transmissíveis e da incapacidade funcional. Rev. Nutr. 2009; 22( 6 ): 937-946.

8. Böhm J, Monteiro MB, Thomé FS. Efeitos do exercício aeróbio durante a hemodiálise em pacientes com doença renal crônica: uma revisão da literatura. J Bras Nefrol. 2012;34(2):189-94.

9. Gusmão L, Galvão J, Possante M. A resposta do rim ao esforc,o físico. Rev Port Nefrol Hipert. 2003;17:73-80.

10. Gomes RJ, Leme JACA, Mello MAR, Luciano E, Caetano FH. Efeitos do treinamento de natação em aspectos metabólicos e morfológicos de ratos diabéticos. Motriz. 2008;14:320-8.

11. Luchi WM, Shimizu MH, Canale D, Gois PH, de Bragança AC, Volpini RA, et al. Vitamin D deficiency is a potential risk factor for contrast-induced nephropathy. Am J Physiol Regul Integr Comp Physiol. 2015;309(3):R215-22.

12. Whiter P., Samson F. E. Determination of inulin in plasm and urine by use of antrone. Journal of Laboratory and Clinical Medicine. 1954;43(3):45-8.

13. Dezoti FC, Watanabe M, Vattimo MFF. Role of heme oxygenase-1 in polymyxin Binduced nephrotoxicity in rats. Antimicrob Agents Chemother. 2012;56(10):5082-7.

14. Kidney Disease: Improving Global Outcomes (KDIGO) CKD Work Group. KDIGO 2012 Clinical Practice Guideline for the Evaluation and Management of Chronic Kidney Disease. Kidney Int. (Suppl) 2013;3(1):5-14.

15. Bastos MG, Kirsztajn GM. Doença renal crônica: importância do diagnóstico precoce, encaminhamento imediato e abordagem interdisciplinar estruturada para melhora do desfecho em pacientes ainda não submetidos à diálise. J Bras Nefrol. 2011; 33(1):93-108.

16. 3. Viana HR, Soares CMBM, Tavares MS, Teixeira MM, Silva ACS. Inflamação na doença renal crônica: papel de citocinas. J Bras Nefrol. 2011;33(3):351-64.

17. Castelao AM, Górriz JL, Bover J, de la Morena JS, Cebollada J, Escalada J, et al. Consensus document for the detection and management of chronic kidney disease. Nefrologia. 2014;34(2):243-62.

18. Castro MCM. Tratamento conservador de paciente com doença renal crônica que renuncia à diálise. J Bras Nefrol. 2019; 41(1):95-102. 
19. World Health Organization (WHO). Global Action Plan 2013 for the prevention and control of noncommunicable diseases 2013-2020. Geneva: WHO; 2013.

20. Nascimento LCA; Coutinho EBS; Silva KNG: Efetividade do exercício físico na insuficiência renal crônica. Fisioter Mov. 2012;25(1):231-9.

21. Kujal P, Vernerová Z. 5/6 nephrectomy as an experimental model of chronic renal failure and adaptation to reduced nephron number. Cesk Fysiol. 2008;57(4):104-9. 